\title{
Cardiovascular magnetic resonance for the detection of descending thoracic aorta calcification in patients with end-stage renal disease
}

\author{
Elbert Edy ${ }^{1 *}+\left(\mathbb{D}\right.$, Alastair J. Rankin ${ }^{1,2+}$, Jennifer S. Lees ${ }^{1,2}$, Pauline Hall Barrientos ${ }^{3}$, Rosemary Woodward ${ }^{4}$, \\ Sokratis Stoumpos ${ }^{1,2}$, loannis Koktzoglou ${ }^{5,6}$, Robert R. Edelman ${ }^{5,7}$, Aleksandra Radjenovic ${ }^{1}$, \\ Patrick B. Mark ${ }^{1,2+}$ and Giles H. Roditi ${ }^{1,8+}$
}

\begin{abstract}
Background: Vascular calcification is an independent predictor of cardiovascular disease in patients with chronic kidney disease. Computed tomography (CT) is the gold-standard for detecting vascular calcification. Radial volumetric-interpolated breath-hold examination (radial-VIBE), a free-breathing gradient-echo cardiovascular magnetic resonance (CMR) sequence, has advantages over CT as it is ionising radiation-free. However, its capability in detecting thoracic aortic calcification (TAC) has not been investigated. This study aims to compare radial-VIBE to CT for the detection of TAC in the descending aorta of patients with end-stage renal disease (ESRD) using semi-automated methods, and to investigate the association between TAC and coronary artery calcification (CAC).

Methods: Paired cardiac CT and radial-VIBE CMR scans from ESRD patients participating in 2 prospective studies were obtained. Calcification volume was quantified using semi-automated methods in a $9 \mathrm{~cm}$ segment of the thoracic aorta. Correlation and agreement between TAC volume measured on CMR and CT were assessed with Spearman's correlation coefficient ( $\rho$ ), linear regression, Bland-Altman plots and intraclass correlation coefficient (ICC). Association between CAC Agatston score and TAC volume determined by CT and CMR was measured with Spearman's correlation coefficient.

Results: Scans from 96 participants were analysed. Positive correlation was found between CMR and CT calcification volume $[p=0.61,95 \%$ confidence interval $(C l) 0.45-0.73]$. ICC for consistency was 0.537 ( $95 \%$ Cl 0.378-0.665). BlandAltman plot revealed that compared to CT, CMR volumes were systematically higher at low calcification volume, and lower at high calcification volume. CT did not detect calcification in $41.7 \%$ of participants, while radial-VIBE CMR detected signal which the semi-quantitative algorithm reported as calcification in all of those individuals. Instances of suboptimal radial-VIBE CMR image quality were deemed to be the major contributors to the discrepancy. Correlations
\end{abstract}

\footnotetext{
*Correspondence: elbertedy@gmail.com

†Elbert Edy and Alastair J. Rankin contributed equally to this manuscript

${ }^{\dagger}$ Patrick B. Mark and Giles H. Roditi are joint senior authors

${ }^{1}$ Institute of Cardiovascular and Medical Sciences, BHF Glasgow

Cardiovascular Research Centre, University of Glasgow, Glasgow G12 8TA,

UK

Full list of author information is available at the end of the article
} permits use, sharing, adaptation, distribution and reproduction in any medium or format, as long as you give appropriate credit to the original author(s) and the source, provide a link to the Creative Commons licence, and indicate if changes were made. The images or other third party material in this article are included in the article's Creative Commons licence, unless indicated otherwise in a credit line to the material. If material is not included in the article's Creative Commons licence and your intended use is not permitted by statutory regulation or exceeds the permitted use, you will need to obtain permission directly from the copyright holder. To view a copy of this licence, visit http://creativecommons.org/licenses/by/4.0/. The Creative Commons Public Domain Dedication waiver (http://creativeco mmons.org/publicdomain/zero/1.0/) applies to the data made available in this article, unless otherwise stated in a credit line to the data. 
between CAC Agatston score and TAC volume measured by CT and CMR were $\rho=0.404$ ( $95 \%$ Cl 0.214-0.565) and $\rho=0.211$ (95\% Cl 0.008-0.396), respectively.

Conclusion: Radial-VIBE CMR can detect TAC with strong positive association to $C T$, albeit with the presence of proportional bias. Quantification of vascular calcification by radial-VIBE remains a promising area for future research, but improvements in image quality are necessary.

Keywords: Thoracic aortic calcification, End-stage renal disease, Cardiovascular disease, Computed tomography, Magnetic resonance imaging, Radial volumetric interpolated breath-hold examination (radial-VIBE) sequence

\section{Background}

Patients with chronic kidney disease (CKD) have a greater risk of cardiovascular disease (CVD) and allcause mortality compared to age-matched controls within the general population [1]. This risk is greatest in patients with end-stage renal disease (ESRD) who require renal replacement therapy in the form of dialysis or a kidney transplant [2]. Traditional cardiovascular risk factors (e.g., diabetes, hypertension, hyperlipidaemia), are prevalent amongst CKD populations. In addition, there are CKD-specific risk factors that contribute to the predisposition to CVD, such as excessive arterial calcification and vascular stiffening. In ESRD patients, aortic stiffness is higher compared to healthy controls [3], and arterial calcification may be partly responsible [4]. Clinically, both arterial stiffness and calcifications have been shown to be independent predictors of CVD mortality in ESRD patients $[5,6]$ and have been used as a surrogate end point for clinical trials in this population [7].

The thoracic aorta can be imaged in both cardiac computed tomography (CT) and cardiovascular magnetic resonance (CMR) and quantifying thoracic aortic calcification (TAC) may have some utility in improving risk prediction. In primary prevention cohort studies, TAC was shown to be an independent predictor for all-cause mortality [8,9], but not for CVD events [10-13]. While in patients with stable angina, TAC was associated with increased risk of death and CVD [14]. Although data in ESRD patients are lacking, other arterial calcifications, such as coronary artery calcification (CAC) and abdominal aortic calcifications, are associated with higher risk of all-cause and cardiovascular mortality in advanced CKD (stages 4-5) and haemodialysis patients [15, 16]. Therefore, it is plausible that TAC might also be a risk factor for CVD events in ESRD patients.

CT is a well-established imaging modality for detecting vascular calcification in clinical practice. Calcification can be quantified on CT using the Agatston score [17], which takes into account the area and density of calcified lesions, or the volume score, which does not depend on calcium density [18]. Traditionally, calcification is hard to discern with conventional spin-echo sequence in CMR because it appears with various signal intensities [19].
Moreover, calcification is hypointense due to low proton density and often lies adjacent to the dark arterial lumen [20].

Recent work has shown that a prototype proton density-weighted in-phase stack-of-stars CMR using a small flip angle gradient-echo readout can accurately quantify aorto-iliac and ilio-femoral vascular calcifications [21, 22]. A similarly configured and commercially-available gradient-echo CMR sequence called radial volumetric interpolated breath-hold examination (radial-VIBE) could therefore serve as a potential alternative to CT in detecting and quantifying vascular calcification without ionising radiation [23]. However, whether this could be applied to detecting TAC is unknown.

The aim of this study was to compare radial-VIBE to CT for the detection and quantification of TAC, specifically in the descending thoracic aorta, in patients with ESRD using a semi-automated approach. In addition, the association between descending TAC and CAC was investigated.

\section{Methods}

\section{Sources of images and other clinical data}

Imaging data from adult patients with ESRD participating in 2 prospective research studies was used: (1) Vitamin $\mathrm{K}$ in kidney transplant organ recipients: Investigating vEssel Stiffness (ViKTORIES) trial (Current Controlled Trials number, ISRCTN22012044) and (2) The Interrogation of the Cardiomyopathy of Chronic Kidney Disease With advancEd caRdiac Imaging (TICKER) study (ClinicalTrials.gov number, NCT03704701). All subjects gave written informed consent to the respective studies, which were reviewed and approved by the local Research Ethics Committee. The ViKTORIES trial [24] is a phase II, double-blinded, parallel-group, randomised, placebocontrolled trial comparing vitamin $\mathrm{K}$ supplementation to placebo on vascular stiffness in renal transplant patients. The TICKER study is an ongoing observational study that is assessing the effects of dialysis on the myocardium using CMR. Both studies collected imaging data, of which the paired cardiac CT and CMR (acquired within $24 \mathrm{~h}$ of each other) were used in the current study. For both VIKTORIES and TICKER studies, the assessment 
of calcification was one of several prospectively defined research questions being addressed. The paired scans were performed at the Clinical Research Imaging Facility based at the Queen Elizabeth University Hospital in Glasgow.

\section{CT image acquisition}

Electrocardiogram (ECG) gated non-contrast scans of the heart were acquired at $120 \mathrm{kVp}$ in a single heartbeat scan using an Aquilion ONE Vision Edition CT scanner (Canon Medical Systems Ltd., Crawley, UK). Radiation dose was reduced by using the Adaptive Iterative Dose Reduction 3D reconstruction algorithm.

\section{CMR image acquisition}

Non-contrast CMR images were obtained using a $3 \mathrm{~T}$ scanner (Prisma, Siemens Healthineers, Erlangen, Germany) with an 18-channel surface coil placed anteriorly, and a 32-channel spine coil placed posteriorly. RadialVIBE images were acquired in coronal plane using the StarVIBE product (Siemens Healthineers), which is a free-breathing, 3D, proton density-weighted, stack-ofstars, gradient echo sequence. The imaging parameters used were: field-of-view (FOV) $462 \times 462 \mathrm{~mm}$, slice thickness $3 \mathrm{~mm}$, repetition time (TR) $4.18 \mathrm{~ms}$, echo time (TE) $2.46 \mathrm{~ms}$, flip angle 2.5 degrees, acquired voxel size $1.2 \times 1.2 \times 1.2 \mathrm{~mm}$, sampling bandwidth $720 \mathrm{~Hz} /$ pixel, scan time $4.30 \mathrm{~min}$. No cardiac or respiratory gating were utilised.

\section{Quantitative image analysis}

One investigator analysed all of the images to measure calcification volume. A second investigator, who was added post-hoc, re-analysed a random sample of scans representing $10 \%$ of the cohort to assess the interobserver reproducibility of our quantitative analysis protocols. To promote blinding, randomly ordered CT images were batch analysed before radial-VIBE images. The latter were analysed in a random order over a week later, without reference to CT results. Horos is a free and open source code software (FOSS) program that is distributed free of charge under the Lesser General Public License at Horosproject.org and sponsored by Nimble Co LLC d/b/a Purview in Annapolis, Maryland, USA. On sagittal views on both CT and radial-VIBE CMR images, Horos was used to select a $9 \mathrm{~cm}$ segment of the descending thoracic aorta, starting from the level of the top of the vertebra closest to the diaphragmatic surface of the heart and then progressing superiorly as shown in Fig. 1. If the vertebra was out of the FOV, then the area of analysis would begin from the inferior surface of the heart. The descending thoracic aorta was chosen as the region of thoracic aorta most reliably visualised on CT within a field of view allowing simultaneous imaging of CAC. The CT and radial-VIBE CMR images containing only the selected portion of aorta were exported and ImageJ (version 1.52q, National Institutes of Health, Bethesda, Maryland, USA) [25] was then used to detect and quantify the volume of calcification present within the descending thoracic aorta. Volume of calcification is the product of the area of the lesions detected and the slice thickness:

$$
\text { Volume } \left._{(\text {in mm }}^{3}\right)=\sum\left(\text { Area in } \mathrm{mm}^{2} * \text { slice thickness in } \mathrm{mm}\right)
$$
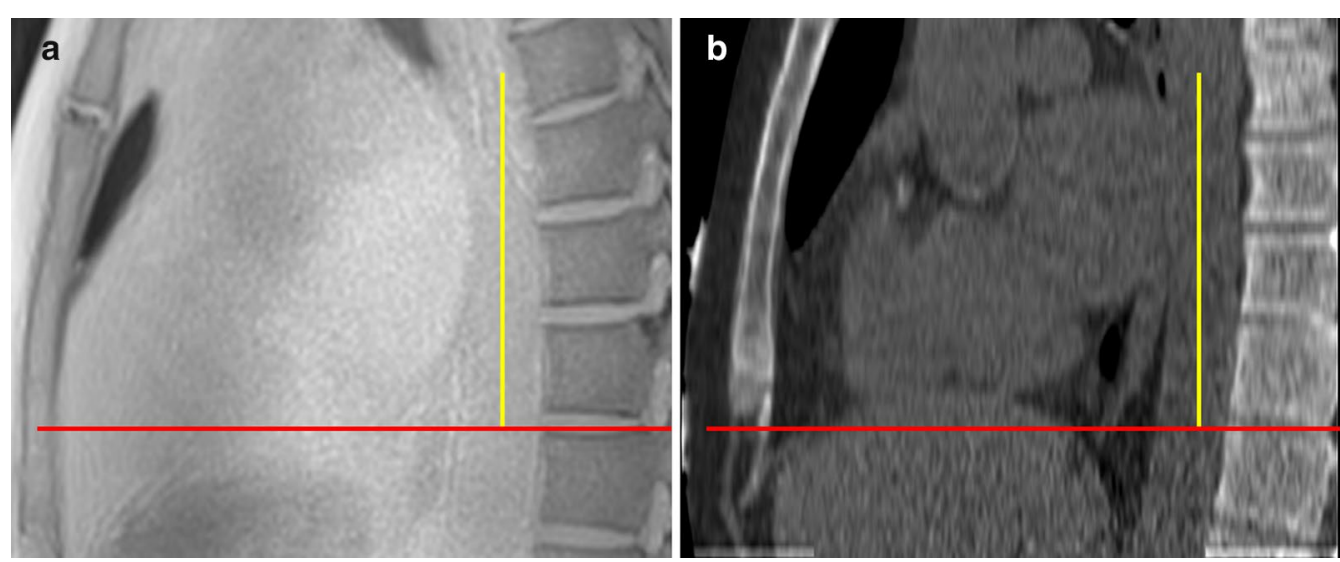

Fig. 1 Sagittal (a) radial-volumetric interpolated breath-hold examination (VIBE) cardiovascular magnetic resonance (CMR) and (b) computed-tomography (CT) images of descending thoracic aorta. A $9 \mathrm{~cm}$ segment of thoracic aorta from the same patient is chosen on both CT and radial-VIBE images. Red horizontal lines mark the level of the top of the vertebra that is closest to the inferior surface of the heart. Yellow vertical lines correspond to $9 \mathrm{~cm}$ of thoracic aorta 


\section{CT image analysis}

CT images were reconstructed with $3 \mathrm{~mm}$ slice thickness and $3 \mathrm{~mm}$ slice interval to be used for analysis. Calcification was defined as voxels with attenuation values of 130 Hounsfield units (HU) or greater and appearing bright on CT (Fig. 2), this corresponds to approximately 2 standard deviations (SD) higher than the attenuation of unenhanced blood. Although arbitrary, this is accepted as the conventional threshold for CT assessment of arterial calcification [17]. A median filter of $3 \mathrm{~mm}$ radius was used to reduce 'salt and pepper' noise while preserving sharp edges. Once a threshold had been set to segment calcifications, a region-of-interest (ROI) was manually drawn around the wall of the descending thoracic aorta if calcified lesion(s) was present. Lastly, the area of calcification within the aorta was measured using automated thresholding. The patient's total volume of calcification was then calculated offline. CAC Agatston scores were reported by a consultant radiologist in line with clinically approved protocols, blinded to treatment allocations and clinical variables using dedicated analysis software (Vitrea Advanced, Vital Images, Minnetonka, Minnesota, USA).

\section{Radial-VIBE image analysis}

Reconstructed radial-VIBE images with $3 \mathrm{~mm}$ slice thickness and $3 \mathrm{~mm}$ slice interval were used for analysis. On the transverse plane, ROIs were manually delineated around the aortic wall in every consecutive slice. Next, a bespoke segmentation algorithm was used to segment calcifications (based on signal intensity) and measure their area. Aortic calcification appears hypointense (i.e., dark) on radial-VIBE images (Fig. 2) and was defined as a voxel with signal intensity of at least 2.5 SD below the
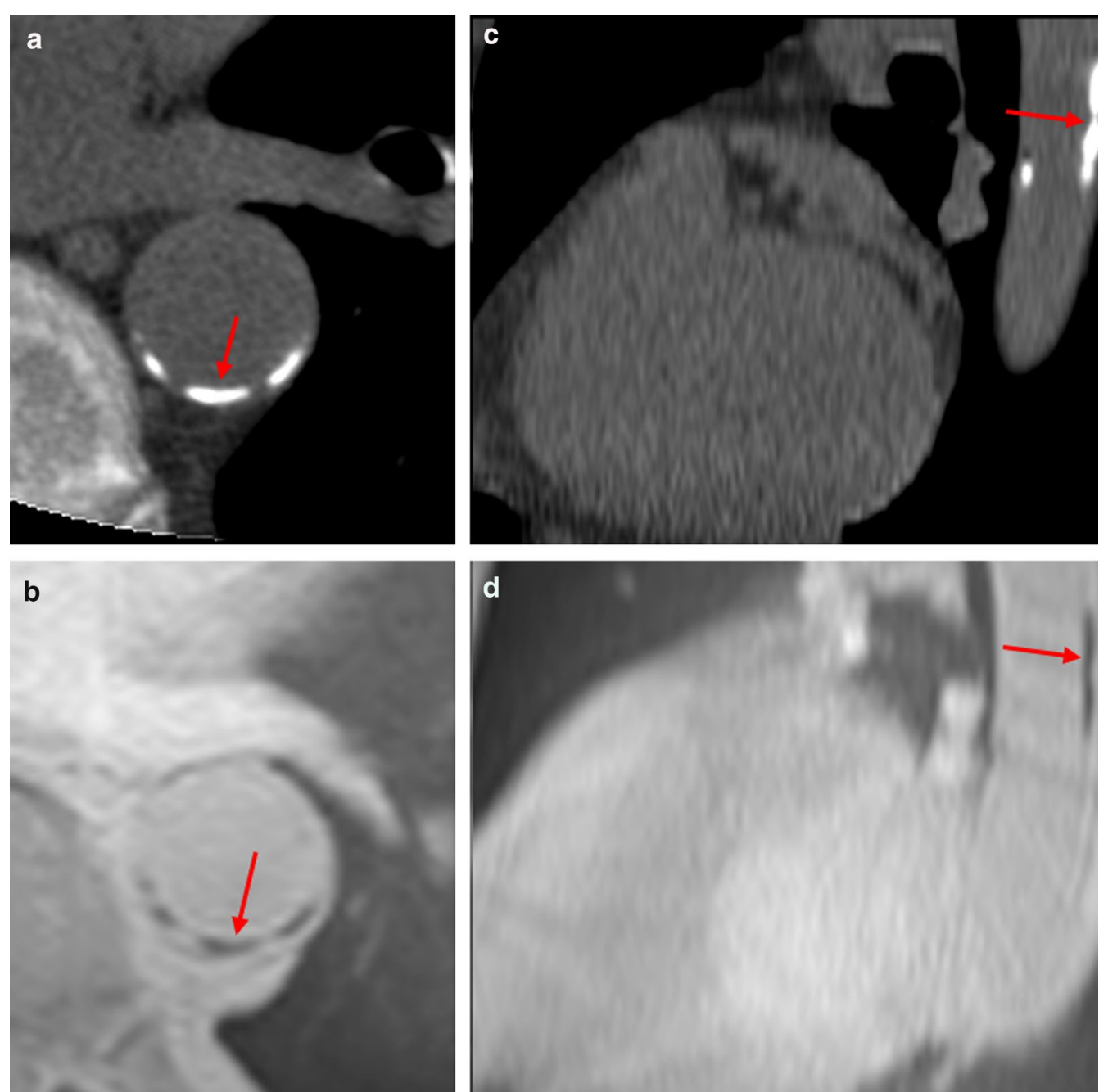

Fig. 2 Representative images of calcifications on CT $(\mathbf{a}, \mathbf{c})$ and radial-VIBE $(\mathbf{b}, \mathbf{d})$. Images $\mathbf{a}$ and $\mathbf{b}$ are axial slices; Images $\mathbf{c}$ and $\mathbf{d}$ are sagittal slices. Calcifications are indicated by the red arrows. For this participant, volume of calcification detected by $C T=835 \mathrm{~mm}^{3}$, radial-VIBE volume $=634 \mathrm{~mm}^{3}$ 
mean signal intensity of voxels within the ROI (i.e., aorta) of each slice. A previous study, which utilised similar techniques, has defined calcification on MRI as between 2 and 3 SD below the mean signal intensity of the ROI [22]. In our cohort, 2.5 SD was chosen as it was quickly evident during the algorithm development that $2 \mathrm{SD}$ insufficiently distinguished calcification from noise, while $3 \mathrm{SD}$ excluded obvious calcification.

\section{Qualitative image analysis}

Two observers (E.E. and A.R., with one and four years experience of researching vascular calcification, respectively) subjectively compared all $(n=96)$ radial-VIBE scans to CT. A 5-point Likert scale (1-very poor, 2poor, 3-fair, 4-good, 5-excellent) was used to assess the confidence with which the radial-VIBE matched the CT with regards to calcification presence, location, size and shape [26]. Where scores disagreed, scans were reviewed to reach consensus.

\section{Statistical analysis}

Data were analysed with Minitab ${ }^{\circledR}$ Statistical Software (version 19.2.0.0, Minitab, State College, Pennsylvania, USA) and SPSS (Statistical Package for the Social Sciences, International Business Machines, Inc., Armonk, New York, USA). Normality of variables was assessed with P-P plot, Kolmogorov-Smirnov, and Shapiro-Wilk tests. Correlation between volume of calcifications in CT and radial-VIBE was assessed with Spearman's rank correlation coefficient $(\rho)$, and linear regression. This is an exploratory, hypothesis-generating, study. The research question was prospectively defined and was considered in the design of the source studies. However, the sample size of the source studies was determined in relation to primary end points unrelated to the methods described in this report. A post-hoc power calculation was not performed.

Spearman's rank correlation coefficient was also used to investigate the association between volume of aortic calcifications (detected by CT and radial-VIBE separately) and CAC Agatston score assessed by CT [27].

Bland-Altman plots were constructed, and mean bias (radial-VIBE volume minus CT volume) and 95\% limits of agreement (LOA) were calculated between CT and radial-VIBE volume. However, as the assumptions of constant mean bias and standard deviation of differences were violated, linear regression was used to model the relationship between mean bias and the mean of calcium volume [28]. The standard deviation of the residuals from regression were then used to estimate $95 \%$ LOA.

To assess the degree of consistency and absolute agreement between $\mathrm{CT}$ and radial-VIBE volume, intra-class correlation coefficient (ICC) estimates and their 95\% confidence interval (CI) based on a single-rating and 2 -way mixed model were calculated. ICC was also used to measure intra- and inter-observer reliability of quantitative analysis by randomly sampling and reanalysing 10 paired CT and radial-VIBE scans after blinding of original volume scores. Weighted Cohen's kappa ( $\mathrm{k}$ ) coefficient was used to assess inter-observer reliability of qualitative scores.

\section{Sensitivity analyses}

As a pre-specified sensitivity analysis, where there was a large discrepancy in the detected calcium volume between radial-VIBE and CT, the area of analysis was reassessed to check for any difference in ROI selection. A re-analysis of the volume of calcification would be done as part of sensitivity analysis if there was a clear discrepancy in area of analysis. This would remove blinding and therefore would be purely exploratory. Data points with standard residuals of 3 or more detected by linear regression were considered as outliers and a sensitivity analysis excluding them was performed.

\section{Results \\ Baseline characteristics}

A total of 96 participants was included in the analysis (24 from TICKER, 72 from ViKTORIES). Twelve were excluded from analysis (1 from TICKER, 11 from ViKTORIES) due to various reasons (See Additional File 1).

The relevant demographic and disease characteristics of the ViKTORIES and TICKER participants are shown in Table 1 . The mean age of study participants was 59.6 years, a third were female and a third were current or ex-smokers (Table 1). Due to the selection criteria chosen for their respective studies, all the TICKER participants were receiving hospital-based intermittent haemodialysis, while ViKTORIES trial participants had a functioning kidney transplant. The median renal replacement therapy vintage was 2 years for TICKER participants and 7 years for ViKTORIES participants (Table 1). The mean estimated glomerular filtration rate (eGFR) for patients with a functioning kidney transplant (i.e., ViKTORIES participants) was $52.5 \mathrm{ml} / \mathrm{min} / 1.73 \mathrm{~m}^{2}$ (SD: $21.8 \mathrm{ml} / \mathrm{min} / 1.73 \mathrm{~m}^{2}$ ).

\section{Radial-VIBE vs CT volume}

The median volume of calcification quantified was 191 $\mathrm{mm}^{3}$ (range 0-1572; IQR $189 \mathrm{~mm}^{3}$ ) by radial-VIBE and $11 \mathrm{~mm}^{3}$ (range 0-4982; IQR $274 \mathrm{~mm}^{3}$ ) by CT. Figure 3 illustrates the scatterplot of volume of TAC measured by radial-VIBE and CT. CT did not detect calcification in a proportion of participants (41.7\%), while radial-VIBE detected signal which the algorithm reported as calcification in all of those individuals (ranging from 65 to 482 
Table 1 Characteristics of TICKER and ViKTORIES participants at baseline

\begin{tabular}{|c|c|c|c|}
\hline Characteristics & ViKTORIES N = 72 & TICKER N $=24$ & Combined $\mathrm{N}=96$ \\
\hline Age, Mean $\pm S D^{*}$ (year) & $57.9 \pm 8.9$ & $64.7 \pm 1.86$ & $59.6(9.3)$ \\
\hline Male sex (\%) & $45(62.8)$ & $15(62.5)$ & $60(66.7)$ \\
\hline White race (\%) & $70(97.2)$ & $21(87.5)$ & $91(94.8)$ \\
\hline Diabetes (\%) & $18(25)$ & $11(45.8)$ & $29(30.2)$ \\
\hline \multicolumn{4}{|l|}{ Smoking status (\%) } \\
\hline Non-smoker & $47(65.3)$ & $18(75.0)$ & $65(67.7)$ \\
\hline Ex-smoker & $19(26.4)$ & $5(20.8)$ & $24(7.3)$ \\
\hline Current smoker & $6(8.3)$ & $1(4.2)$ & $7(25.0)$ \\
\hline Previous cardiovascular disease $^{\dagger}$ & $17(23.6)$ & $11(45.8)$ & $28(29.2)$ \\
\hline $\mathrm{eGFR}$, mean $\pm \mathrm{SD}\left(\mathrm{ml} / \mathrm{min} / 1.73 \mathrm{~m}^{2}\right)^{\ddagger}$ & $52.5 \pm 21.8$ & - & - \\
\hline \multicolumn{4}{|c|}{ Renal replacement therapy vintage (years) } \\
\hline Median & 7.10 & 1.96 & - \\
\hline Interquartile range & 10.48 & 2.69 & - \\
\hline
\end{tabular}

*SD denotes standard deviation

+ Participants were considered to have previous cardiovascular disease if they had one or more of the following: history of ischaemic heart disease, heart failure, coronary revascularisation (including percutaneous coronary intervention and/or coronary artery bypass graft), stroke and/or transient ischaemic attack, and/or peripheral arterial disease

${ }^{\ddagger}$ eGFR denotes estimated glomerular filtration rate

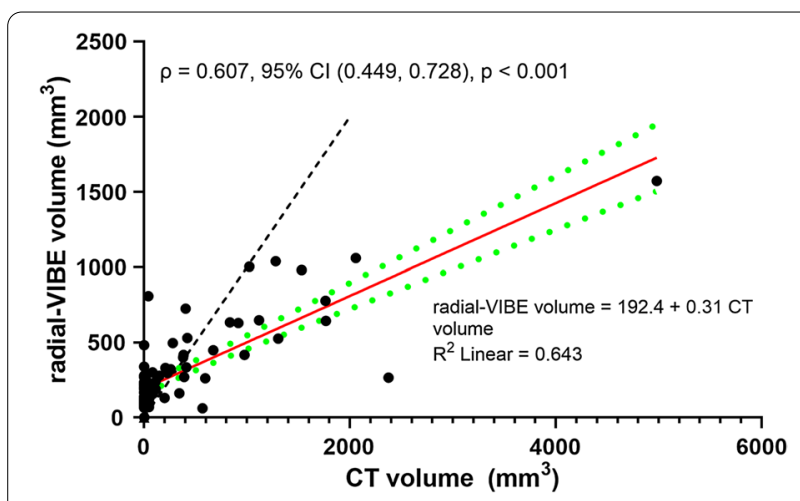

Fig. 3 Scatterplot of thoracic aortic calcification volume measured by radial-VIBE against CT. Red solid line is the line of best fit and green dashed lines represent its 95\% confidence intervals (Cl); black dashed line is the line of unity. The linear regression equation and R-squared value are on the bottom right; Spearman's rank correlation coefficient $(\rho)$, with its $95 \% \mathrm{Cl}$ and $\mathrm{p}$-value, are on the top left

$\mathrm{mm}^{3}$ ). There was only one case when calcification was detected in CT $\left(2.2 \mathrm{~mm}^{3}\right)$ and none in radial-VIBE.

Spearman's rank correlation coefficient was assessed as variables were not normally distributed. There was a positive monotonic correlation between radial-VIBE and CT calcification volume $[\rho=0.607,95 \%$ CI $(0.449,0.728)$, $\mathrm{p}<0.001]$. Linear regression equation for radial-VIBE volume was $192.4+0.31 \mathrm{CT}$ volume.

The ICC estimates based on single-measure and 2-way mixed effects model for consistency and absolute agreement were similar [ICC for consistency: $0.537,95 \% \mathrm{CI}$

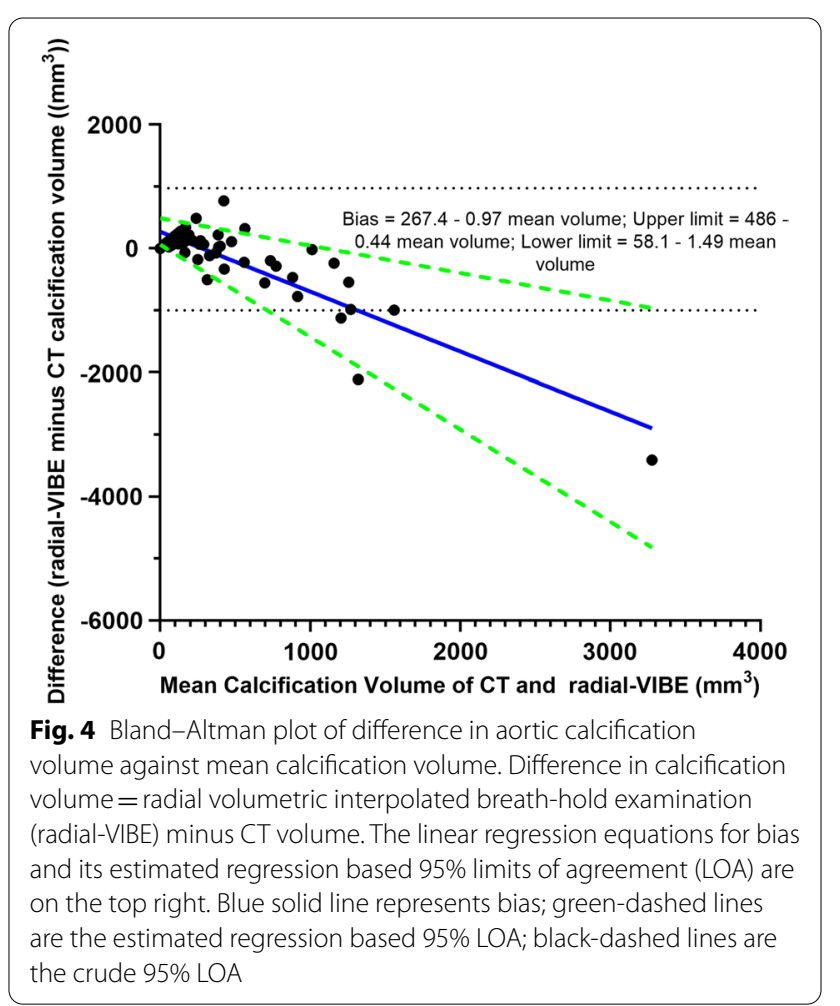

$(0.378,0.665)$; ICC for absolute agreement: $0.539,95 \% \mathrm{CI}$ $(0.380,0.667)]$.

According to the Bland-Altman plot (Fig. 4), the bias (radial-VIBE volume minus CT volume) and standard deviations of the differences were proportional to the 
magnitude of mean volume (i.e., proportional bias was present). At lower calcification volume, differences tend to be positive (i.e., radial-VIBE values were higher than $\mathrm{CT})$. As the mean volume of calcification detected by $\mathrm{CT}$ and radial-VIBE increased, radial-VIBE values decreased proportionally relative to $\mathrm{CT}$ values and the bias became increasingly negative.

The relationship between bias and mean calcium volume modelled with linear regression was: Bias $=267.4-$ 1.0 mean volume. P-value of the slope $(-0.967)$ was $<0.001$, thus confirming that the difference in volume is related to the magnitude of volume (i.e., mean volume). The 95\% LOA could be visualised in Fig. 4, which illustrates that the standard deviations of the differences between radial-VIBE and CT results were also related to the mean volume.

\section{Intra- and inter-observer reliability}

The intra-observer reliability based on absolute agreement and 2-way mixed effects model was 1.00 for CT and 0.993 [95\% CI $(0.957,0.998), \mathrm{p}<0.001$ ] for radial-VIBE measurements. The inter-observer reliability using the same measures was 1.00 for CT and 0.990 [95\% CI (0.959, 0.997), $\mathrm{p}<0.001$ ] for radial-VIBE.

\section{Comparison with coronary artery agatston score}

There was a positive association between TAC volume detected by CT and CAC Agatston score [ $\rho=0.404,95 \%$ CI $(0.214,0.565), \mathrm{p}<0.001]$. Meanwhile, there was also a positive, but weaker, association between TAC measured by radial-VIBE and CAC Agatston score $[\rho=0.211,95 \%$ CI $(0.008,0.396), \mathrm{p}=0.039$ ].

\section{Outliers and sensitivity analyses}

Three participants were identified as outliers as their standard residuals were larger than 3 . The sections of aorta that were analysed on the CT and radial-VIBE in these patients were reviewed and deemed to be similar. Radial-VIBE images from 2 of the patients were of poor quality and had obvious artefact, which was likely to have influenced the results. No obvious explanation was found for the other outlier. Sensitivity analyses were performed with exclusion of these 3 outliers and the results remained relatively unchanged (results, scatter plot and Bland-Altman plot included in Additional files 2, 3).

\section{Qualitative assessment}

The mean Likert score for the subjective, qualitative assessment of radial-VIBE compared to CT was 4.6 (SD 0.8 ). There was good agreement between the individual scores from the 2 observers $[\kappa=0.822,95 \%$ CI $(0.717$, 0.927), $\mathrm{p}<0.001]$. For the subgroup of 40 participants in whom quantitative analysis showed falsely detected 'calcification' on radial-VIBE that was not present on $\mathrm{CT}$, the mean Likert score was 4.6 (SD 0.9). A total of 31 (78\%) of these scored excellent agreement for no calcification being present, while 8 were downgraded for small volume false-positive findings on radial-VIBE, with one also having a small volume of false negative. In one participant, radial-VIBE correctly identified a small area of calcification that was present on the CT, but which was subsequently removed from the $\mathrm{CT}$ images when the median filter was applied for quantitative analysis. Figure 5 shows case examples of when the subjective assessment of the paired scans yielded excellent agreement but the semi-automated, quantitative algorithm resulted in both over-detection and under-detection of calcification.

\section{Discussion}

This study confirms that radial-VIBE is an option to detect and quantify TAC with a positive association when compared to the gold standard CT. On subjective assessment radial-VIBE performs well when compared to CT, but when quantifying volume using a semi-automated method, the association is imperfect and proportional bias is observed. Compared to CT, radial-VIBE over-estimates the volume of calcification when minimal calcification is present and under-estimates it when extensive calcification is present.

At lower mean volume of calcium, radial-VIBE had systematically higher volume of TAC lesions than CT. Several reasons might be responsible for this discrepancy. Firstly, it might be due to the presence of noise on radial-VIBE images, which had been falsely detected as calcification (Fig. 5). The application of a median filter to reduce noise on radial-VIBE images was attempted but it affected the detection of obvious calcium lesions as well. On the other hand, a median filter was applied on the CT images, which could have contributed to the difference. Smaller calcified lesions were possibly removed by the filter, and hence explain the over-estimation of calcification volume when low levels of calcification were present, with one definite instance of this discovered on qualitative assessment. As an exploratory post-hoc analysis, we re-analysed the 40 participants who had TAC on radialVIBE but not on CT. Removing the CT filter for these participants did not produce a meaningful improvement in results (data not shown). It is also possible that radialVIBE images were affected by the presence of other compounds (e.g., haemosiderin) undetected by $\mathrm{CT}$, which resulted in the presence of susceptibility artefacts. Due to the use of a proton density-weighted in-phase acquisition providing a bland image contrast, the boundaries of the aortic wall were visually obscured on most radial-VIBE scans. This made accurate delineation of the aorta challenging. It was likely that non-aortic voxels were included 

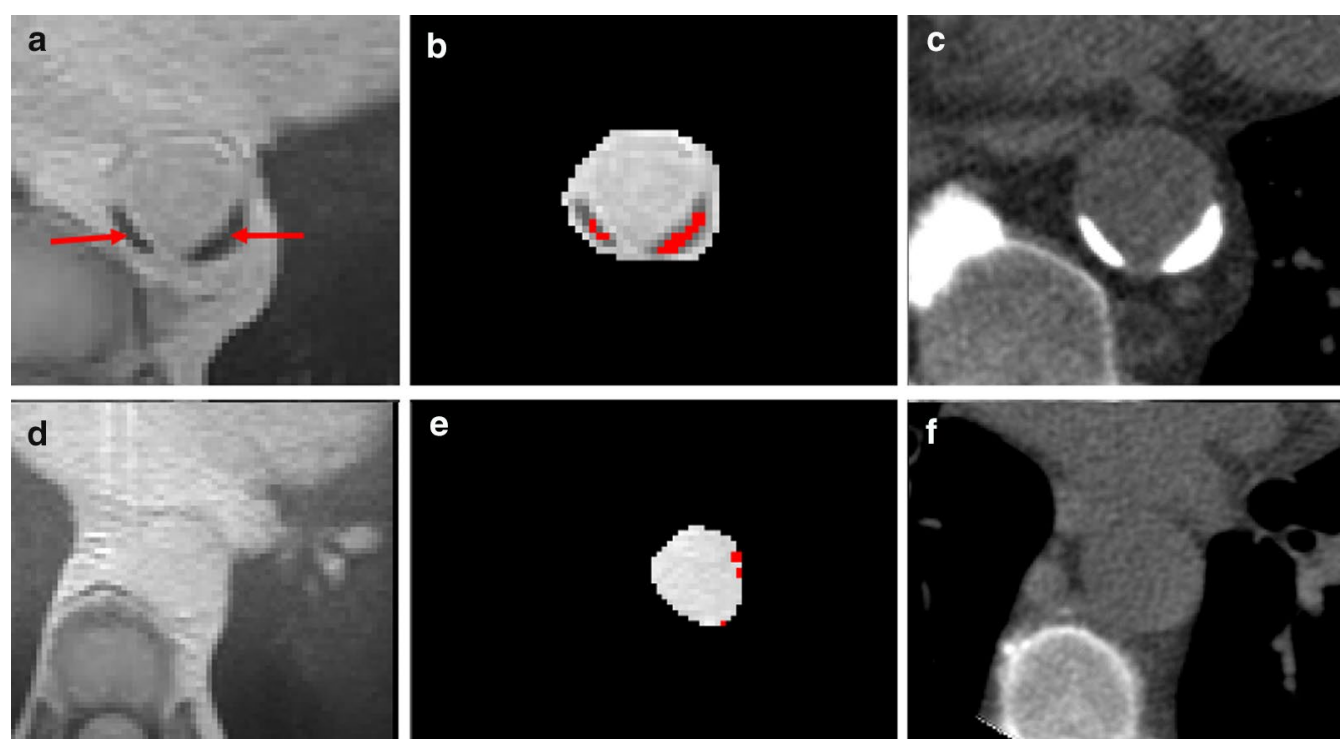

Fig. 5 Representative images of under-detection $(\mathbf{a}, \mathbf{b})$ and over-detection $(\mathbf{d}, \mathbf{e})$ on radial-VIBE compared to CT. Images $\mathbf{a}$ and $\mathbf{b}$ are axial slices of radial-VIBE and image $\mathrm{C}$ is an axial slice of CT from the same patient. Red arrows on images $\mathbf{a}$ and $\mathbf{b}$ indicate calcifications, which appear as hypointense voxels. Red areas on image $\mathbf{b}$ illustrate the voxels that are considered as calcifications by the segmentation algorithm. Images $\mathbf{d}$ and $\mathbf{e}$ are identical axial slices of radial-VIBE belonging to another patient, and image $\mathrm{F}$ is the corresponding CT slice. Red areas on image e are the voxels considered as calcifications by the segmentation algorithm, which are likely to be noise and not genuine calcifications. For these two patients, the subjective analysis deemed excellent agreement between radial-VIBE and CT, despite the quantitative analysis differing significantly

as ROI, which could introduce more noise and affect the segmentation algorithm's calculation of the threshold. Lastly, this study used a substantially larger voxel size than reported in a prior study of aorto-iliac and ilio-femoral calcifications [22], so that partial volume averaging of the low-signal calcifications with surrounding tissues may have been a more significant issue than in the prior study. Until improvements in radial-VIBE image quality are realised, a stepped analysis approach in which a subjective assessment is performed prior to algorithmic quantification of calcium deposits may negate some issues surrounding the false positive rate observed in the present study.

At higher mean volumes of calcium, radial-VIBE volumes were smaller than $\mathrm{CT}$ volumes. The reason for this could be due to blooming artefact of calcifications on $\mathrm{CT}$, inappropriately magnifying dense lesions resulting in overestimation of their volume [29]. However, this assumes vascular calcification is more susceptible to blooming artefact on CT than CMR, which may not be true. Another factor could be the presence of noise within the aorta of some radial-VIBE images that could cause uneven signal intensity and inflate the standard deviation (Fig. 5). Consequently, the threshold for calcification would be extremely high and thus, diminish the segmentation algorithm's ability to detect calcification. This was found to be the case for one of the outliers that was identified, and another one in which radial-VIBE did not pick up any calcification but the paired CT did. The same effect could also occur when very large, dense areas of calcification are present, such that the calcium signal impacts on the mean intensity of the vessel lumen and subsequently, the threshold for calcification.

This study has several strengths. Firstly, the scanning protocols were standardised to limit variability. Secondly, $\mathrm{CT}$ and radial-VIBE images analysed in this study were obtained within $24 \mathrm{~h}$ of each other, so the amount of calcification present in the patient's thoracic aorta would be identical during the acquisition of both scans. Thirdly, by studying patients with ESRD, who have a higher vascular calcium burden than their age- and sex-matched controls [30], we could compare calcium quantification across a range of severity (as evident by the present results showing CT calcium volume ranging from 0 to $4982 \mathrm{~mm}^{3}$ ). The present findings would be applicable to other patient groups with a propensity towards vascular calcification including those with diabetes [31], CVD [30] and the elderly [32].

\section{Limitations}

One of the limitations of this project was the manual delineation of aorta in the analysis of radial-VIBE images, whereas CT images were analysed with a more automated approach. Besides the poor definition of 
aortic wall in radial-VIBE images impairing the accuracy of manual segmentation as previously mentioned, manually drawing ROI was time-consuming and was less reproducible than $\mathrm{CT}$ analysis. However, several reasons led to that decision. Unlike CT, where the Hounsfield unit is calibrated with reference to water and its values are comparable among patients, the absolute signal intensity in CMR is not. The signal intensity is affected by various factors, which include proton density, pulse sequence, types and strength of magnet used for scanning [33]. Thus, the magnitude of signal intensity of the same tissue (e.g., heart) may vary across individuals. Consequently, it meant that calcium has no 'absolute' value which we could use for thresholding and segmentation. Instead, thresholding calcification in CMR images was dependent on its relative signal intensity compared to surrounding voxels-hence we used signal intensity greater than 2.5 SD below the mean signal intensity of ROI. This approach for thresholding calcifications in CMR images was used by Serhal et al. in their study, where 2 and 3 SD below the mean were classified as calcifications in the aortoiliac and femoral arteries, respectively [22]. Due to the poor image quality of some scans in this study (often due to loss of signal from the large field of view/participant body habitus), 2 SD was not used as it could not sufficiently distinguish calcifications from noise, while $3 \mathrm{SD}$ would significantly impair the detection of calcium with relatively low signal intensity. Furthermore, the thoracic aorta is near the air-filled lungs which are also hypointense. Segmenting the aorta while avoiding the lungs was crucial in order to prevent the mistake of identifying voxels in the lungs as calcification. Initially, the creation of a mask of the aorta from HASTE (Half-Fourier-Acquired Single-shot Turbo spin-Echo) images to segment the aorta was explored. Unlike radial-VIBE images, HASTE images have good aortic definition and manual segmentation is easier. Unfortunately, it was not possible to use the HASTE mask to subtract surrounding tissues from the radial-VIBE images due to different image parameters on the acquired scans (e.g., slice thickness and resolution).

Another limitation was that the typical CT scans for CAC do not cover the aortic arch and proximal descending thoracic aorta, which made it more challenging to find a common anatomical landmark in CT and radialVIBE images to ensure that similar segments of the aorta were analysed. Additionally, the aortic arch and proximal descending thoracic aorta have been shown to be the areas of the aorta most prone to calcification [34] and it would have been useful to compare the detection of calcification by radial-VIBE and CT in those aortic segments.

The discrepancy in TAC volume between radial-VIBE and $\mathrm{CT}$ suggests that $\mathrm{CT}$ should remain the primary modality for assessing vascular calcification in clinical practice. Furthermore, CT has the advantages of wider availability, lower cost, and faster acquisition time compared to radial-VIBE. However, there is no doubt that radial-VIBE can detect vascular calcification and, if improvements in image quality are realised, it may be a plausible alternative to $\mathrm{CT}$ in the future, particularly where patients are undergoing CMR angiography studies where additional information on calcified plaque can be valuable. The near-future role of radial-VIBE is perhaps best suited to realm of research, where its lack of ionising radiation can allow serial imaging, either as part of longitudinal study trying to monitor the progression of vascular calcification, or to assess the impact of therapeutics in clinical trials. Radial-VIBE has the additional benefit of allowing other CMR sequences to be acquired, thus providing information on the possible effects of therapies on cardiac structure and function, as well as aortic distensibility. However, ensuring adequate image quality would be essential to allow accurate quantification of TAC using radial-VIBE sequence.

\section{Conclusions}

This study supports the hypothesis that radial-VIBE can detect TAC. However, there is proportional bias in the measurement of calcium volume by radial-VIBE compared to CT. Quantification of vascular calcification by radial-VIBE remains a promising area for future research, but improvements in image quality (e.g., through the use of optimised protocols, smaller voxels and motion correction) are necessary.

\section{Abbreviations}

CAC: Coronary artery calcification; Cl: Confidence interval; CKD: Chronic kidney disease; CMR: Cardiovascular magnetic resonance; CT: Computed tomography; CVD: Cardiovascular disease; eGFR: Estimated glomerular filtration rate; ESRD: End-stage renal disease; FOSS: Free and open source code software; FOV: Field-of-view; HASTE: Half-Fourier-Acquired Single-shot Turbo spin-Echo; HU: Hounsfield units; ICC: Intraclass correlation coefficient; IQR: Interquartile range; LOA: Limits of agreement; Radial-VIBE: Radial volumetric-interpolated breath-hold examination; ROI: Region-of-interest; SD: Standard deviation; TAC :Thoracic aortic calcification; TE: Echo time; TICKER: The Interrogation of the Cardiomyopathy of Chronic Kidney Disease With advancEd caRdiac Imaging; TR: Repetition time; ViKTORIES: Vitamin K in kidney transplant organ recipients: investigating vEssel stiffness.

\section{Supplementary Information}

The online version contains supplementary material available at https://doi. org/10.1186/s12968-021-00769-6.

Additional file 1. Flowchart of cardiovascular magnetic resonance (CMR) and computed tomography (CT) scans available for analysis.

Additional file 2. Sensitivity Analysis (with 3 outliers excluded) Results and Scatterplot.

Additional file 3. Sensitivity Analysis Bland-Altman plot. 


\section{Acknowledgements}

We wish to thank the participants for their contribution to this research. We wish to thank the radiographers at the Clinical Research Facility, Queen Elizabeth University Hospital, Glasgow, UK, for their expertise in image acquisition.

\section{Authors' contributions}

All authors have reviewed and contributed to this manuscript. AJR, JSL, SS, PHB, GHR and PBM conceived the study idea and study design. AJR and JSL recruited participants. RW led image acquisition. EE performed image analysis and statistical analysis. EE and AJR wrote the manuscript. IK wrote an initial version of software used for CMR calcium segmentation. IK, AR, RE and GHR advised on image analysis and critically reviewed the manuscript. SS and PBM critically reviewed the manuscript. All authors read and approved the final manuscript.

\section{Funding}

Dr Alastair Rankin is personally funded by a Clinical Academic Training Fellowship from The Chief Scientist Office (Scotland) (CAF/18/02). Dr Jennifer Lees was personally funded by a Kidney Research UKTraining Fellowship (TF_013_20161125) for the ViKTORIES study and was supported by a British Heart Foundation Centre of Excellence Award (RE/13/5/30177) and Chief Scientist Office (Scotland) Lectureship (PCL/20/10). The investigators decided the study design, data collection, analysis, interpretation and presentation of results with no input from the study funders.

\section{Availability of data and materials}

Source data will be provided upon reasonable request.

\section{Declarations}

\section{Ethics approval and consent to participate}

The West of Scotland Research Ethics Committee granted ethical approval for the acquisition and analysis of the images obtained from the TICKER and ViKTORIES trials. REC reference number for the TICKER study: 18/WS/0138. REC reference number for the ViKTORIES trial: 17/WS/0101. Written informed consent was provided by all participants.

\section{Consent for publication}

Written informed consent was provided for all representative images.

\section{Competing interests}

The authors declare no competing interests in relation to the present study. Dr. Tim Leiner served as the JCMR Guest Editor for this manuscript.

\section{Author details}

${ }^{1}$ Institute of Cardiovascular and Medical Sciences, BHF Glasgow Cardiovascular Research Centre, University of Glasgow, Glasgow G12 8TA, UK. ${ }^{2}$ Renal and Transplant Unit, Queen Elizabeth University Hospital, Glasgow, UK. ${ }^{3}$ Department of Clinical Physics and Bioengineering, NHS Greater Glasgow and Clyde, Glasgow, UK. ${ }^{4}$ Clinical Research Imaging, NHS Greater Glasgow and Clyde, Glasgow, UK. ${ }^{5}$ Radiology, NorthShore University HealthSystem, Evanston, IL 60201, USA. ${ }^{6}$ Radiology, University of Chicago Pritzker School of Medicine, Chicago, IL, USA. ${ }^{7}$ Radiology, Feinberg School of Medicine, Northwestern University, Chicago, IL, USA. ${ }^{8}$ Department of Radiology, NHS Greater Glasgow and Clyde, Glasgow, UK.

Received: 18 September 2020 Accepted: 4 May 2021

Published online: 24 June 2021

\section{References}

1. Gansevoort RT, Correa-Rotter R, Hemmelgarn BR, Jafar TH, Heerspink HJL, Mann JF, et al. Chronic kidney disease and cardiovascular risk: epidemiology, mechanisms, and prevention. The Lancet. 2013;382(9889):339-52.

2. Kidney disease: improving global outcomes (KDIGO) CKD Work Group. KDIGO 2012 clinical practice guideline for the evaluation and management of chronic kidney disease. Kidney Int Suppl. 2013;3(1):1-150.

3. Zimmerli LU, Mark PB, Steedman T, Foster JE, Berg GA, Dargie HJ, et al. Vascular function in patients with end-stage renal disease and/or coronary artery disease: a cardiac magnetic resonance imaging study. Kidney Int. 2007;71(1):68-73.

4. Guérin AP, London GM, Marchais SJ, Metivier F. Arterial stiffening and vascular calcifications in end-stage renal disease. Nephrol Dial Transplant. 2000;15(7):1014-21.

5. Blacher J, Guerin AP, Pannier B, Marchais SJ, London GRM. Arterial calcifications, arterial stiffness, and cardiovascular risk in end-stage renal disease. Hypertension. 2001;38(4):938-42.

6. Blacher J, Guerin AP, Pannier B, Marchais SJ, Safar ME, London GRM. Impact of aortic stiffness on survival in end-stage renal disease. Circulation. 1999;99(18):2434-9.

7. Raggi P, Bellasi A, Bushinsky D, Bover J, Rodriguez M, Ketteler M, et al. Slowing progression of cardiovascular calcification with SNF472 in patients on hemodialysis. Circulation. 2020;141(9):728-39.

8. Allison MA, Hsi S, Wassel CL, Morgan C, Ix JH, Wright CM, et al. Calcified atherosclerosis in different vascular beds and the risk of mortality. Arteriosclerosis, Thrombosis, Vasc Biol. 2012;32(1):140-6.

9. Santos RD, Rumberger JA, Budoff MJ, Shaw LJ, Orakzai SH, Berman D, et al. Thoracic aorta calcification detected by electron beam tomography predicts all-cause mortality. Atherosclerosis. 2010;209(1):131-5.

10. Budoff MJ, Nasir K, Katz R, Takasu J, Carr JJ, Wong ND, et al. Thoracic aortic calcification and coronary heart disease events: the multi-ethnic study of atherosclerosis (MESA). Atherosclerosis. 2011;215(1):196-202.

11. Hoffmann U, Massaro JM, D'Agostino RB, Kathiresan S, Fox CS, O'Donnell CJ. Cardiovascular event prediction and risk reclassification by coronary, aortic, and valvular calcification in the Framingham heart study. J Am Heart Assoc. 2016;5(2):e003144.

12. Mahabadi AA, Lehmann N, Möhlenkamp S, Pundt N, Dykun I, Roggenbuck $U$, et al. Noncoronary measures enhance the predictive value of cardiac CT above traditional risk factors and CAC score in the general population. JACC Cardiovasc Imaging. 2016;9(10):1177-85.

13. Wong ND, Gransar H, Shaw L, Polk D, Moon JH, Miranda-Peats R, et al. Thoracic aortic calcium versus coronary artery calcium for the prediction of coronary heart disease and cardiovascular disease events. JACC Cardiovasc Imaging. 2009;2(3):319-26.

14. Eisen A, Tenenbaum A, Koren-Morag N, Tanne D, Shemesh J, Imazio M, et al. Calcification of the thoracic aorta as detected by spiral computed tomography among stable angina pectoris patients. Circulation. 2008;118(13):1328-34.

15. Cano-Megías M, Guisado-Vasco P, Bouarich $H$, de Arriba-de la Fuente G, de Sequera-Ortiz P, Álvarez-Sanz C, et al. Coronary calcification as a predictor of cardiovascular mortality in advanced chronic kidney disease: a prospective long-term follow-up study. BMC Nephrol. 2019;20(1):188.

16. Okuno S, Ishimura E, Kitatani K, Fujino Y, Kohno K, Maeno Y, et al. Presence of abdominal aortic calcification is significantly associated with all-cause and cardiovascular mortality in maintenance hemodialysis patients. Am J Kidney Dis. 2007;49(3):417-25.

17. Agatston AS, Janowitz WR, Hildner FJ, Zusmer NR, Viamonte M, Detrano R. Quantification of coronary artery calcium using ultrafast computed tomography. J Am Coll Cardiol. 1990;15(4):827-32.

18. Blaha MJ, Mortensen MB, Kianoush S, Tota-Maharaj R, Cainzos-Achirica M. Coronary artery calcium scoring. JACC Cardiovasc Imaging. 2017;10(8):923-37.

19. Tsuchiya K, Makita K, Furui S, Nitta K. MRI appearances of calcified regions within intracranial tumours. Neuroradiology. 1993;35(5):341-4.

20. Koktzoglou I. Gray blood magnetic resonance for carotid wall imaging and visualization of deep-seated and superficial vascular calcifications. Magnetic Reson Med. 2013;70(1):75-85.

21. Ferreira Botelho MP, Koktzoglou I, Collins JD, Giri S, Carr JC, Gupta N, et al. MR imaging of iliofemoral peripheral vascular calcifications using proton density-weighted, in-phase three-dimensional stack-of-stars gradient echo. 2016

22. Serhal A, Koktzoglou I, Aouad P, Carr JC, Giri S, Morcos O, et al. Cardiovascular magnetic resonance imaging of aorto-iliac and ilio-femoral vascular calcifications using proton density-weighted in-phase stack of stars. J Cardiovasc Magnetic Reson. 2018;20(1).

23. Stoumpos S, Hall Barrientos P, Black DH, Stevenson K, Hennessy M, Vesey AT, et al. Ferumoxytol MR angiography: a novel technique for assessing iliac vasculature in potential kidney transplant recipients. JACC Cardiovasc Imaging. 2020;13(8):1847-8. 
24. Lees JS, Mangion K, Rutherford E, Witham MD, Woodward R, Roditi G, et al. Vitamin $\mathrm{K}$ for kidney transplant organ recipients: investigating vessel stiffness (ViKTORIES): study rationale and protocol of a randomised controlled trial. Open Heart. 2020;7(2):e001070.

25. Schneider CA, Rasband WS, Eliceiri KW. NIH Image to ImageJ: 25 years of image analysis. Nat Methods. 2012;9(7):671-5.

26. Likert R. A technique for the measurement of attitudes. New York: The Science Press; 1932.

27. Cohen J. A power primer. Psychol Bull. 1992;112(1):155-9.

28. Bland JM, Altman DG. Measuring agreement in method comparison studies. Stat Methods Med Res. 1999;8(2):135-60

29. Hoffmann U, Ferencik M, Cury RC, Pena AJ. Coronary CT angiography. J Nucl Med. 2006;47(5):797-806.

30. Braun J, Oldendorf M, Moshage W, Heidler R, Zeitler E, Luft FC. Electron beam computed tomography in the evaluation of cardiac calcifications in chronic dialysis patients. Am J Kidney Dis. 1996;27(3):394-401.
31. Schurgin S, Rich S, Mazzone T. Increased prevalence of significant coronary artery calcification in patients with diabetes. Diabetes Care. 2001;24(2):335.

32. Allison MA, Criqui MH, Wright CM. Patterns and risk factors for systemic calcified atherosclerosis. Arterioscler Thromb Vasc Biol. 2004;24(2):331-6.

33. Bloem JL, Reijnierse M, Huizinga TWJ, Van Der Helm-Van Mil AHM. MR signal intensity: staying on the bright side in MR image interpretation. RMD Open. 2018;4(1):e000728.

34. Craiem D, Chironi G, Casciaro ME, Graf S, Simon A. Calcifications of the thoracic aorta on extended non-contrast-enhanced cardiac CT. PLoS ONE. 2014;9(10):e109584.

\section{Publisher's Note}

Springer Nature remains neutral with regard to jurisdictional claims in published maps and institutional affiliations.
Ready to submit your research? Choose BMC and benefit from:

- fast, convenient online submission

- thorough peer review by experienced researchers in your field

- rapid publication on acceptance

- support for research data, including large and complex data types

- gold Open Access which fosters wider collaboration and increased citations

- maximum visibility for your research: over 100M website views per year

At BMC, research is always in progress.

Learn more biomedcentral.com/submissions 